\title{
Hermansky-Pudlak Syndrome 2
}

National Cancer Institute

\section{Source}

National Cancer Institute. Hermansky-Pudlak Syndrome 2. NCI Thesaurus. Code C150368.

An autosomal recessive sub-type of Hermansky-Pudlak syndrome caused by mutation(s) in the AP3B1, encoding AP-3 complex subunit beta-1. Immunodeficiency due to neutropenia is a characteristic of this sub-type. 